\title{
EL GÉNERO Undastriatostyliolina EN LA CORDILLERA CANTÁBRICA (NO DE ESPAÑA). SISTEMÁTICA, EVOLUCIÓN Y PALEOBIOGEOGRAFÍA
}

\author{
Montse TRUYOLS-MASSONI \\ Departamento de Geología, Universidad de Oviedo. C/ J. Arias de Velasco s/n. \\ 33005-OVIEDO.
}

Truyols-Massoni, M. 1999. El género Undastriatostyliolina en la Cordillera Cantábrica (NO de España). Sistemática, evolución y paleobiogeografía. [The genus Undastriatostyliolina in the Cantabrian Mountains (NW Spain). Systematics, evolution and palaeobiogeography.] Revista Española de Paleontología, $\mathbf{n}^{\mathbf{0}}$ extr. Homenaje al Prof. J. Truyols, 239-249. ISSN 0213-6937.

\begin{abstract}
Several Undastriatostyliolina species are described for the first time from an area outside the Boulonnais (N France), the type area of the genus. Three are new species: Undastriatostyliolina truyolsi n. sp., Undastriatostyliolina n. sp. 3, and Undastriatostyliolina n. sp. 1. Two of these, U. truyolsi and U. n. sp. 3, occur in upper Givetian strata, together with $U$. globicellata. Finding the other new species, Undastriatostyliolina $\mathrm{n}$. sp. 1, in Emsian strata allows postulating the likely origin of the family Undastriatostyliolinidae, and permits analysing the evolutionary trends in this family. The oldest species, Undastriatostyliolina $\mathrm{n}$. sp. 1, has a larger sized shell than the other species in this genus. Therefore, contrary to the usual tendency in most of the invertebrate groups, the dacryoconarids seem to show a trend towards smaller forms in younger horizons. Thus, in most of the dacryoconarid lineages the following general tendencies can be observed: 1) a progresive decrease in shell size; 2) a progresive decrease in the number of longitudinal elements; 3) a diminution in prominence of the transverse rings. These general tendencies in the dacryoconarids are also reflected in the genus Undastriatostyliolina. Some palaeobiogeographic information is provided as well. On the available data, the genus Undastriatostyliolina seems to have originated during late Emsian times in an area presently situated in the Cantabrian Mountains. It was at that time when provincialism diminished on both sides of the Rheic Ocean, so that planktonic forms, with small shells, could apparently move easily from one place to another, being able to migrate at a good place over considerable distances.
\end{abstract}

Keywords: Dacryoconarids, Undastriatostyliolinids, Cantabrian Mountains, systematics, new species, Emsian, Givetian, evolutionary trends, palaeobiogeography.

\section{RESUMEN}

Varias formas de Undastriatostyliolina son descritas por vez primera fuera de la región tipo del género. Tres son especies nuevas, Undastriatostyliolina truyolsi n. sp., Undastriatostyliolina n. sp. 3 y Undastriatostyliolina n. sp. 1. Las dos primeras, junto con Undastriatostyliolina globicellata, aparecen en el Givetiense Superior. El hallazgo de Undastriatostyliolina n. sp. 1 en capas emsienses, ha permitido establecer el probable origen de la Familia Undastriatostyliolinidae y analizar sus posibles tendencias evolutivas. Undastriatostyliolina n. sp. 1, la especie más antigua, posee una concha de tamaño mucho mayor que las demás formas conocidas del género. Aunque un fenómeno habitual en la evolución de diferentes grupos de invertebrados es el aumento de tamaño del cuerpo, los dacrioconáridos parecen mostrar una tendencia evolutiva contraria. Así en la mayoría de linajes, es posible generalizar unas tendencias evolutivas globales en el sentido de mostrar una disminución progresiva en el tamaño de las conchas y en el número de elementos longitudinales así como una aminoración del relieve en la ornamentación transversal. Y esta tendencia general parece repetirse en el caso del género Undastriatostyliolina. Se aportan también algunas consideraciones paleobiogeográficas de interés. En el estado actual de conocimientos, el origen del género Undastriatostyliolina se encuentra en la Cordillera Cantábrica, en el Emsiense Superior. En esta época comienza a disminuir el provincialismo en muchas regiones a ambos lados del Océano Reico, de modo que, organismos planctónicos con conchas de pequeño tamaño, debieron poseer una alta 
capacidad de desplazamiento y pudieron superar distancias importantes, tal vez sin excesivas difícultades, lo que permitió su rápida dispersión.

\section{Palabras clave: Dacrioconáridos, Undastriatostiliolínidos, Cordillera Cantábrica, sistemática, especies nuevas, Emsiense, Givetiense, tendencias evolutivas, paleobiogeografía.}

\section{INTRODUCCIÓN}

El Dominio Palentino constituye una pequeña área de afloramientos silúricos y devónicos situados al SE de la Zona Cantábrica (Fig. 1A). Esta Zona, así como las restantes del Macizo Ibérico (de acuerdo con la división de Julivert et al., 1972), presenta su propia historia dinámica y sedimentaria. Aunque en todas ellas el Devónico está variablemente representado, las Zonas Cantábrica y Asturoccidental-Leonesa son las únicas que conservan un registro sedimentario prácticamente continuo.

Las particulares características sedimentológicas, paleontológicas y estructurales de los dispersos afloramientos devónicos del Dominio Palentino han permitido su interpretación (Frankenfeld, 1983; Marquínez y Marcos, 1984; Rodríguez-Fernández y Heredia, 1990, entre otros) como una unidad alóctona correspondiente a una porción de la Zona Asturoccidental-Leonesa, deslizada durante la orogenia herciniana, hacia el NE, sobre la Zona Cantábrica, interpretación que se asume en este trabajo. Desde un punto de vista estratigráfico, García-Alcalde et al. (1990a) aportaron una información sintética y concisa de la región, describiendo de forma resumida sus diferentes unidades litoestratigráficas, mostrando no sólo la distribución de los principales taxones, sino también las biozonas basadas en diferentes grupos orto y paracronológicos y, consecuentemente, sus límites cronoestratigráficos.

En conjunto el Devónico cantábrico está constituido en su mayor parte por materiales siliciclásticos entre los que se intercalan episodios carbonatados de carácter arrecifal que debieron depositarse en medios neríticos de escasa profundidad (magnafacies mixta). En cambio, la sucesión devónica palentina la forman fundamentalmente lutitas carbonatadas y calizas arcillosas nodulosas que corresponden a depósitos alejados de la costa, no necesariamente profundos, pero sí de aguas tranquilas, propios de la magnafacies bohemoherciniana. Por ello, la fauna más representativa de la región está constituida por formas nectónicas y planctónicas, tales como ammonoideos, dacrioconáridos y homocténidos, aunque es frecuente, no obstante, la presencia en muchos de sus niveles de una rica fauna de braquiópodos, ostrácodos, trilobites y conodontos, así como la esporádica presencia de algunos rugosos solitarios y de ciertas formas de tabulados.

El conocimiento de los dacrioconáridos de la Península Ibérica es relativamente reciente. Aunque existen citas aisladas en el contexto de diversos trabajos de índole sistemática, es sólo a partir de los años 70 cuando su conocimiento adquiere cierto interés por sus importantes aplicaciones bio y cronoestratigráficas. En este sentido, la mayor parte de los trabajos han dado prioridad a destacar las formas de mayor interés estratigráfico (Truyols-Massoni, 1981 a y b, 1986; Montesinos y Truyols-Massoni, 1987; Truyols-Massoni en García Alcalde et al. 1990a y b; Truyols-Massoni en García-López et al. 1990; García-Alcalde y TruyolsMassoni, 1994, entre otros), relegándose a un segundo plano la descripción de otros taxones menos conocidos, pero no por ello carentes de importancia. El objetivo de este trabajo es, justamente, poner en evidencia varias especies nuevas del género Undastriatostyliolina en el Dominio Palentino, ya que este taxón había sido reconocido hasta ahora únicamente en su región tipo del Boulonnais, al N de Francia (Farsan, 1983). Más tarde, Farsan (1994) mencionó la presencia de su nueva Familia Undastriatostyliolinidae no sólo en Francia, sino también en Canadá, Marruecos y China, aunque su presencia se registra, en esas regiones, por medio de otros taxones distintos de Undastriatostyliolina.

\section{SITUACIÓN ESTRATIGRÁFICA}

El género Undastriatostyliolina aparece representado con relativa abundancia en la Formación Cardaño. Esta unidad, de unos 80 metros de espesor, está constituida por calizas nodulosas grises, calizas margosas y margas, alternando con niveles de lutitas oscuras, que se hacen más frecuentes en la parte alta, y que contienen con frecuencia horizontes con nódulos carbonatados. Por medio de su contenido fósil, representado por ammonoideos, braquiópodos, homocténidos y conodontos, se le ha podido asignar una edad Givetiense

Figura 1. A.- Esquema de la Zona Cantábrica y parte de la Asturoccidental-Leonesa, en la que se han diferenciado los materiales silúrico-devónicos de la Región del Pisuerga-Carrión (Dominio Palentino), donde se localizan las sucesiones estudiadas. B.- Columna estratigráfica parcial de la Fm. Abadía en la sección Arauz con la situación de los niveles muestreados y la distribución de los diferentes dacrioconáridos. La sección se localiza en la hoja 81 (Potes) a escala 1:50.000, coordenadas UTM: x: 369700 e y: 4762500, del mapa geológico de España. C.- Columna estratigráfica parcial de la Fm. Cardaño en la sucesión del río Carrión con los niveles muestreados y la distribución de los diferentes dacrioconáridos. La sección se localiza en la margen derecha del río Carrión, próxima a su confluencia con el arroyo El Calero, al ENE del pueblo de Vidrieros (coordenadas geográficas $4^{\circ} 36^{\prime}$ ' $20^{\prime \prime} \mathrm{O}$ y $42^{\circ}$ 57' 16" N de la hoja 106, "Camporredondo de Alba", del mapa geológico de España a escala 1:50.000). 


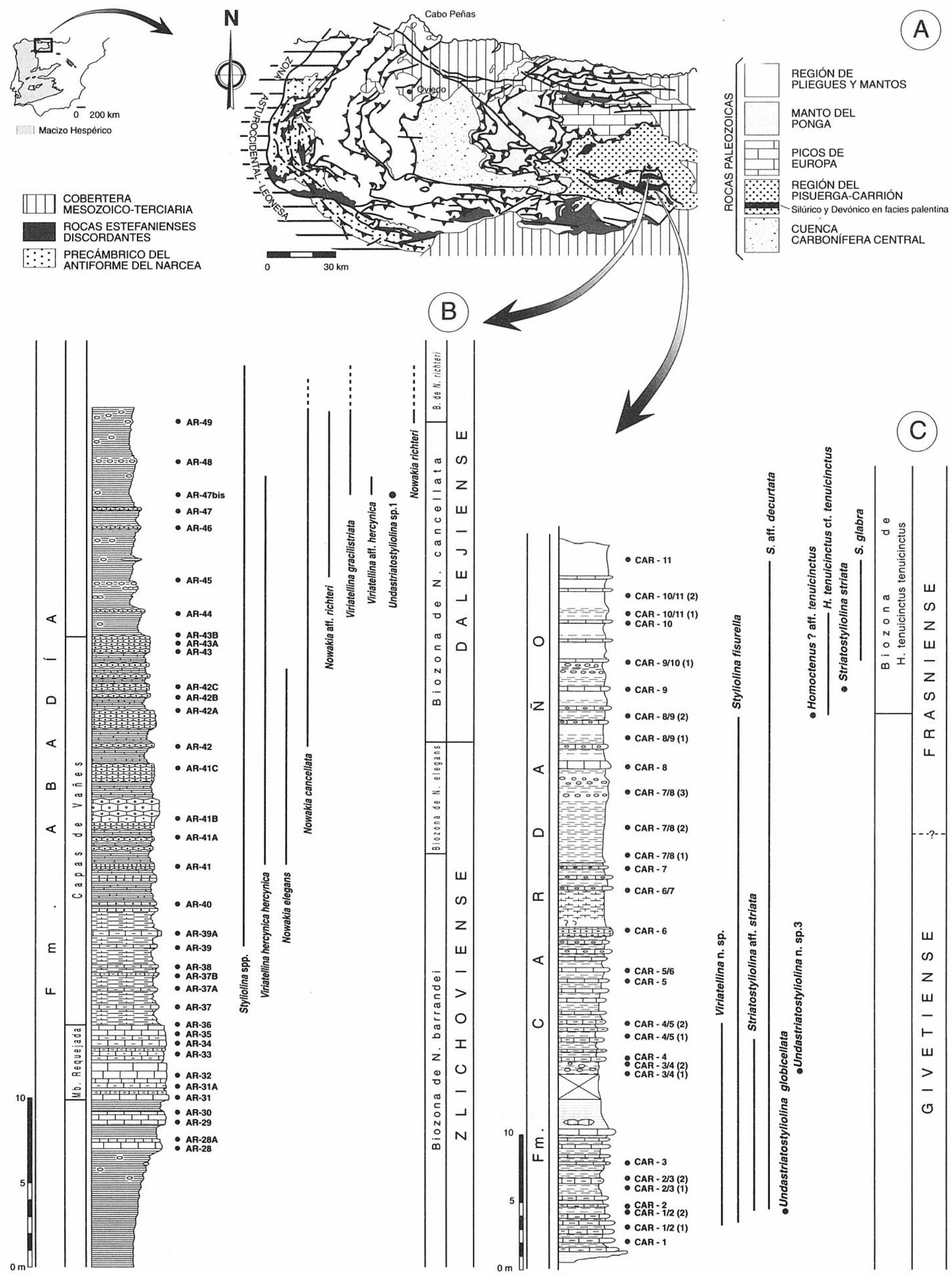


Superior-Fameniense Inferior (Jahnke et al., 1983; Montesinos y Henn, 1986; García-Alcalde et al. 1990a; Montesinos y Sanz López, 1997, entre otros). Los tramos que han suministrado diversas formas del género Undastriatostyliolina pertenecen exclusivamente al Givetiense Superior, dentro de la Biozona de conodontos de Polygnathus varcus (Henn, 1985) y por debajo de capas con Schindewolfoceras alcaldei, especie que aparece en un intervalo estratigráfico situado entre las biozonas de conodontos de Schmidtoganthus hermanniPalmatolepis cristatus y Polyganthus asymmetricus Más Baja, según Montesinos y Henn (1986).

Hasta la actualidad, Undastriatostyliolina ha sido encontrada únicamente en dos secciones de la Fm. Cardaño: la sucesión Gleithang (Henn, 1985) y la sucesión del río Carrión (Truyols-Massoni, 1988), probablemente equivalente a la sección Pralhang de Henn (1985) y parcialmente a Cardaño I de Montesinos y Henn (1986). En la primera, bien situada y descrita por Henn (op cit., p.75), la autora de este trabajo ha podido determinar Undastriatostyliolina truyolsi $\mathrm{n}$. $\mathrm{sp}$. procedente de los niveles 39 y 43 y Undastriatostyliolina globicellata Farsan, del 43. Todas las muestras de dacrioconáridos recolectadas por Henn (1985) se encuentran actualmente depositadas en la Universidad de Oviedo y han sido reestudiadas por la autora. Ambos taxones habían sido asignados por Henn (op. cit. p. 75) a Striatostyliolina sp. y/o Viriatellina sp.

La segunda sección (Truyols-Massoni, 1988), se describe en lo que sigue (Fig. 1C). Situada en la margen derecha del río Carrión, próxima a su confluencia con el arroyo El Calero, al ENE del pueblo de Vidrieros (coordenadas geográficas $4^{\circ} 36^{\prime} 20^{\prime \prime} \mathrm{O}$ y $42^{\circ} 57^{\prime} 16^{\prime \prime} \mathrm{N}$ de la hoja 1:50.000 de Camporredondo de Alba), la sucesión representa un afloramiento de la parte inferior de la Formación Cardaño. Las capas más bajas visibles por encima del nivel habitual del río (tramo CAR-1/2(2)) han suministrado $U$. globicellata Farsan, mientras que una nueva especie, Undastriatostyliolina n. sp. 3, aparece en un tramo ligeramente más moderno (CAR-3/4(1)).

Se ha encontrado, no obstante, otra especie de Undastriatostyliolina en capas mucho más antiguas. En efecto, un único ejemplar bien distinto, como se verá mas adelante, de todas las especies conocidas del género, proviene de capas emsienses de la Fm. Abadía. Esta formación comprende una sucesión de unos $150 \mathrm{~m}$ de espesor, de lutitas oscuras, areniscas, margas, calizas y limolitas. La parte inferior está constituida por lutitas oscuras con una importante intercalación arenosa. Sobre ella se desarrolla un conjunto calcáreo (Mb. Requejada), seguido de unas alternancias de lutitas carbonatadas y margas (Capas de Vañes) a las que se superponen términos limolíticos. La unidad culmina con niveles de lutitas calcáreas y margas (Mb. Lezna). La edad de la formación ha podido ser determinada, por medio de una rica fauna de conodontos, dacrioconáridos, ammonoideos, braquiópodos, corales y trilobites, como Emsiense, aunque los tramos inferiores de la misma pertenecen aún al Praguiense (García-Alcalde et al., 1990a y b, entre otros). La nueva especie de este género,
Undastriatostyliolina n. sp. 1, ha sido encontrada hasta el momento únicamente en la sección Arauz, en un nivel de la Fm. Abadía situado unos $25 \mathrm{~m}$ por encima del Mb. Requejada. En la Fig. 1 se representan los niveles alrededor de este hallazgo; la serie completa puede consultarse en Truyols-Massoni (1988). Una parte de esta sección, correspondiente a los niveles en torno al $\mathrm{Mb}$. Requejada, fue estudiada por Kullmann (1960). Más recientemente, Montesinos (1991) describió de nuevo esta misma sucesión, pero algo más completa, incluyendo unos $35 \mathrm{~m}$ por encima de las calizas del Miembro Requejada. La columna representada en la Fig. $1 \mathrm{~B}$ de este trabajo es comparable a la descrita por Montesinos (op. cit., fig. 6) de modo que el nivel de donde procede Undastriatostyliolina n. sp. 1 debe ser el mismo o muy próximo al que ha suministrado a Montesinos (op. cit.) Mimagoniatites ex gr. fecundus. Undastriatostyliolina n. sp. 1 está acompañada de otros dacrioconáridos significativos, como Viriatellina hercynica hercynica Boucek, V. cf. gracilistriata Hall, Nowakia aff. richteri Boucek y Prantl y, en especial, Nowakia cancellata R. Richter. Los primeros representantes de $N$. richteri aparecen $4,5 \mathrm{~m}$ por encima de la capa con la nueva especie, lo que permite confirmar que Undastriatostyliolina $\mathrm{n}$. sp. 1 aparece en la parte superior de la Biozona de dacrioconáridos de $N$. cancellata, es decir, en el Emsiense Superior. Esta sección ha sido recientemente estudiada por TruyolsMassoni (en prensa), poniendo en evidencia la distribución de los ammonoideos en relación con las biozonas de dacrioconáridos y con los principales eventos geo-biológicos emsienses.

\section{EVOLUCIÓN Y PALEOBIOGEOGRAFÍA}

Como se ha dicho anteriormente, las únicas formas conocidas en la actualidad del género Undastriatostyliolina procedían de la región del Boulonnais (norte de Francia), en concreto, de la parte media de la Formación Beaulieu, dentro del Miembro Pâtures (Farsan, 1983). Al Miembro Pâtures se le ha podido asignar una edad Frasniense, dentro de la Biozona de conodontos de Po. asymmetricus Media (Brice, 1988). Esto significa que las formas francesas han sido encontradas en niveles más jóvenes que los tramos cantábricos con mayor abundancia de undastriatostiliolínidos, esto es, las capas givetienses de la Fm. Cardaño.

Sin embargo, como se ha indicado con anterioridad, el primer representante del género es aún mucho más antiguo. En efecto, Undastriatostyliolina n. sp. 1 procede de un nivel de la Fm. Abadía que pertenece a la Biozona de dacrioconáridos de $N$. cancellata, del Emsiense superior.

Aunque un fenómeno generalizado en la evolución de diferentes grupos de invertebrados es el aumento de tamaño del cuerpo, los dacrioconáridos, y probablemente también los homocténidos, parecen mostrar una tendencia evolutiva contraria. En efecto, principalmente entre los dacrioconáridos, parece constante que un carácter tal 
como el gran tamaño relativo de las conchas, unido a un fuerte relieve de la ornamentación de anillos transversales, junto con un elevado número de elementos longitudinales, son propios de formas primitivas en diversas líneas filogenéticas. De este modo, parece posible generalizar unas tendencias evolutivas globales en el sentido de mostrar una disminución progresiva en el tamaño de las conchas y en el número de elementos longitudinales así como una aminoración del relieve en la ornamentación transversal. Esto es especialmente evidente entre los dacrioconáridos en el linaje $N$. barrandei- $N$. richteri, tendencias a las que se unen un aumento progresivo del tamaño de los tubérculos, juntamente con una aparición cada vez más temprana de la ornamentación. Pero este fenómeno puede también constatarse en otras líneas evolutivas del grupo. Así, por ejemplo, en las líneas filogenéticas $N$. acuaria- $N$. praecursor- $N$. sulcata y $N$. acuaria- $N$. zlichovensis- $N$. karpinskii, de Lütke (1979) se manifiesta una tendencia a la ligera reducción del tamaño de las conchas, unida a una clara disminución del número de costillas longitudinales y a la aminoración del relieve de los elementos transversales.

Esta tendencia general del grupo parece repetirse también en el caso del género Undastriatostyliolina. En efecto, la forma más antigua del grupo, del Emsiense, posee una concha de tamaño muy superior, casi el doble, que la de las formas givetienses ibéricas y las frasnienses del Boulonnais. Asimismo, puede observarse una clara tendencia a la pérdida de relieve de los anillos transversales y a la disminución del número de estrías, tal vez con la única excepción de la especie Undastriatostyliolina n. sp. 3.

Es tal vez un poco prematuro avanzar conclusiones demasiado firmes en relación con la filogenia de Undastriatostyliolina, dado el poco conocimiento que aún se posee del género y su escasa presencia, reducida tan solo a dos áreas. Sin embargo, en ausencia de nuevos datos que pongan de manifiesto la presencia de estas formas en otras regiones devónicas, parece que el origen del género Undastriatostyliolina debiera buscarse en el Dominio Palentino de la Cordillera Cantábrica, en niveles del Emsiense superior. En esta época comienza a disminuir el provincialismo en muchas regiones, facilitado por las importantes vías de comunicación abiertas no sólo entre diversas regiones del norte del continente gondwánico, sino también entre esta masa continental y la región sur de Báltica, lo que favorecería la buena dispersión de sus faunas. Tal como señala García-Alcalde (1995), parece constatarse que en el Emsiense, las faunas bentónicas, fundamentalmente de trilobites, corales y braquiópodos (véase Arbizu, 1978; Soto, 1979; Racheboeuf, 1981 y Morzadec et al., 1988) ponen en evidencia notorias semejanzas entre la región cantábrica, el macizo armoricano, el norte de África y la región apalachense.

En el dominio pelágico, las faunas de dacrioconáridos parecen concordar con los datos anteriores, aunque resulta destacable la ausencia de faunas pelágicas de dacrioconáridos en el dominio apalachense con anterioridad al Emsiense, tal como fue señalado por Lütke (1985). Sin embargo, a partir del Devónico Medio, su presencia en esta región es ya un hecho. En efecto, Lütke (1985) ya constató la existencia de formas comunes entre Nevada y las áreas bohémica, armoricana, cantábrica y norteafricana a partir del Emsiense, pero también con la costa este de Norteamérica (Nueva York, Ohio, etc.), mientras que las formas praguienses y lochkovienses de dacrioconáridos parecen mostrar aún un cierto provincialismo entre ambos dominios, como sucede con las faunas bentónicas. En este mismo sentido, Farsan (1994) señaló la presencia de la Familia Undastriatostyliolinidae, con dudas, ya desde el Devónico Medio, no sólo en Europa y norte de África sino también en Norteamérica, en concreto Canadá, aunque sin precisar localidades.

De este modo es posible suponer que, a pesar de las recientes reconstrucciones paleogeográficas debidas a Bergström (1990) y Scotese y McKerrow (1990), el Océano Reico debió ser bastante más estrecho de lo supuesto, lo que facilitó la existencia de importantes vías de comunicación, tal como ya han señalado diversos autores (Bouyx et al., 1992; García-Alcalde, 1993 y 1995, entre otros). Ciertamente, en épocas más modernas, givetienses y frasnienses, el acercamiento de los grandes continentes gondwánico y americano (Laurentia+Báltica) favoreció aún más las comunicaciones y, por tanto, el cosmopolitismo. De hecho, Brice et al. (1994) muestran las afinidades existentes entre las faunas marinas bentónicas del Dominio del Viejo Mundo y de los Apalaches. Sin embargo, organismos con conchas de muy pequeño tamaño y con un hábito de vida planctónico, debieron poseer una capacidad de desplazamiento muy superior a las formas bentónicas, y habrían podido superar distancias importantes tal vez sin excesivas dificultades. Con sus delicadas conchas, no obstante, los dacrioconáridos en su conjunto debieron estar condicionados por la existencia de aguas no muy agitadas, tal vez mares poco profundos con plataformas bien oxigenadas, lo que permitió su rápida dispersión.

Con todo lo dicho, los hallazgos de Undastriatostyliolina, hasta ahora tan sólo en la región francesa del Boulonnais y en el Dominio Palentino de la Península Ibérica, no deberían ser casos aislados, y su ausencia en variadas regiones de ambos dominios paleogeográficos es, sin duda, consecuencia de una falta de datos por la carencia de exploración. A pesar de ello, parece estar en la Cordillera Cantábrica el origen del grupo, donde han sido encontradas las formas más antiguas de undastriatostiliolínidos. No obstante, nueva información sobre su hallazgo en otras áreas permitiría establecer un entramado evolutivo mucho más sólido.

\section{SISTEMÁTICA}

Todo el material estudiado se encuentra depositado en el Departamento de Geología de la Universidad de Oviedo (DPO-34077 a 34086 ambos inclusive). Una parte del mismo ha sido recolectado por la autora (sección Arauz y 
sección del río Carrión) (Fig. 1). El resto procede de la sucesión "Gleithang", descrita por Henn (1985, p. 75) para la realización de su tesis doctoral. La colección de dacrioconáridos muestreada por este autor (Henn, op. cit.) fue revisada por la autora y trasladada al Departamento de Geología de Oviedo para su depósito. El material se encuentra en un variable estado de conservación. Los ejemplares procedentes de tramos lutíticos suelen conservarse en estado de moldes, generalmente deformados durante el proceso de compactación. En cambio, los especímenes provenientes de los niveles carbonatados están menos deformados y, a menudo, conservan su concha, aunque con frecuencia alterada superficialmente y, en ocasiones, decalcificada.

\section{Familia Undastriatostyliolinidae Farsan,1994}

Género-tipo: Undastriatostyliolina Farsan, 1983.

\section{Diagnosis}

Conchas cónicas, con ornamentación longitudinal de estrías y elementos esculturales transversales en forma de tenues ondulaciones y/o surcos. Conchas de 10 milimicras de grosor. Para detalles menores véase Farsan (1994).

Discusión: Véase Farsan (1983, 1994).

Géneros: Undastriatostyliolina Farsan, Distriatostylus Lardeux, Sinoviriatellina Li, Hemipsila Mu y Mu.

Distribución estratigráfica (enmendada aquí): Devónico Inferior-Frasniense Superior.

Género Undastriatostyliolina Farsan,1983

Especie-tipo: U. curvimarginata Farsan,1983.

\section{Diagnosis}

Conchas pequeñas, cuyo ángulo de crecimiento varía poco a lo largo de la ontogenia, de manera que presentan, en general, forma cónica. Ornamentación transversal consistente en ondulaciones muy poco pronunciadas. Ornamentación longitudinal constituida por finas estrías de densidad variable. Cámara inicial normalmente mal individualizada.

\section{Discusión}

Este género fue incluido por su autor (Farsan, 1983) en la Familia Striatostyliolinidae por la presencia de estrías longitudinales. Más tarde, él mismo (Farsan, 1994) creó una nueva familia a la que incorporó otros tres géneros ya conocidos, Distriatostylus, Sinoviriatellina y Hemipsila.

La posesión de elementos ornamentales transversales claros, aunque débiles, indicaría que el género está probablemente relacionado con la Familia Nowakiidae. No hay que olvidar, sin embargo, que Undastriatostyliolina fue previamente asignado a la Familia Striatostiliolinidae, familia con la que, sin duda, mantiene también relaciones de parentesco. Metastyliolina Boucek y Prantl incluido entre los estriatostiliolínidos, muestra, en ocasiones, suaves ondulaciones transversales en la proximidad de la apertura. Así pues, Undastriatostyliolina comparte afinidades tanto con los nováquidos como con los estriatostiliolínidos. De hecho, se aproxima al nováquido Viriatellina Boucek por sus similares elementos transversales, pero mostrando estrías en lugar de costillas, y al estriatostiliolínido Striatostyliolina Boucek y Prantl por la presencia de estrías longitudinales. Esto confirma la unidad del grupo de los dacrioconáridos, cuyos diferentes planes estructurales están enlazados por morfologías intermedias tales como las del género que discutimos.

El género Undastriatostyliolina ha sido reconocido por primera vez, a nuestro conocimiento, fuera de su

\section{Lámina I}

1,2 Undastriatostyliolina sp. 1. Fm. Abadía, nivel 47 de la sección Arauz, Biozona de N. cancellata. DPO 34077. 1. (40x). Látex de un molde externo casi completo; 2. (55x) Detalle de la región adulta próxima a la apertura donde se observan las estrías bien marcadas.

3 - 5 Undastriatostyliolina truyolsi n. sp. Fm. Cardaño. 3, 4. (80x) DPO 34082 y 34083, respectivamente. Paratipos procedentes del nivel 39 de la sección Gleithang (Henn, 1985, p. 75); látex de moldes externos casi completos, salvo la cámara inicial. 5. (80x) DPO 34081. Holotipo procedente del nivel 43 de la sección Gleithang (Henn, 1985, p. 75) en el que se observa la densa ornamentación transversal y las profundas estrías longitudinales; látex de molde externo completo.
6, 7, 9 Undastriatostyliolina sp. 3. Fm. Cardaño, nivel CAR-3/4(1) de la sección Carrión. 6. (80x) DPO 34084. Látex de molde externo de una región adulta muy deformada por aplastamiento. 7. (80x) DPO 34085. Fragmento de un ejemplar muy desgastado; las estrías son visibles en la parte proximal del ejemplar. 9. (80x) DPO 34086. Fragmento aplastado distalmente; como en el especimen anterior, las estrías se observan mejor en su parte proximal.

8, 10, 11 Undastriatostyliolina globicellata Farsan, 1983. Fm. Cardaño. 8, 10. (80x) DPO 34078 y 34079, respectivamente. Látex de moldes externos casi completos procedentes de la sección Gleithang, nivel 43; obsérvese que las estrías recubren la cámara inicial. 11. (80x) DPO 34080. Látex de un molde externo sin cámara inicial ni la parte distal de la región adulta, procedente del nivel CAR1/2(2) de la sección Carrión. 


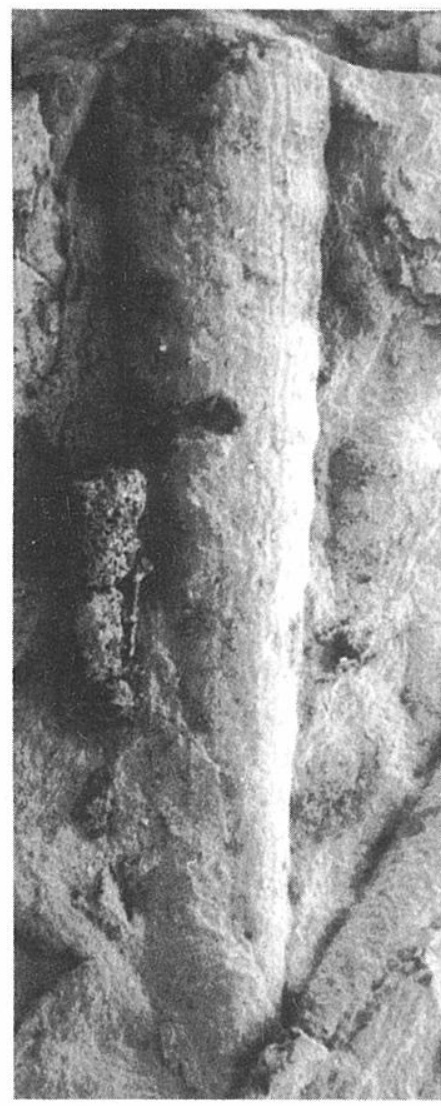

1

5

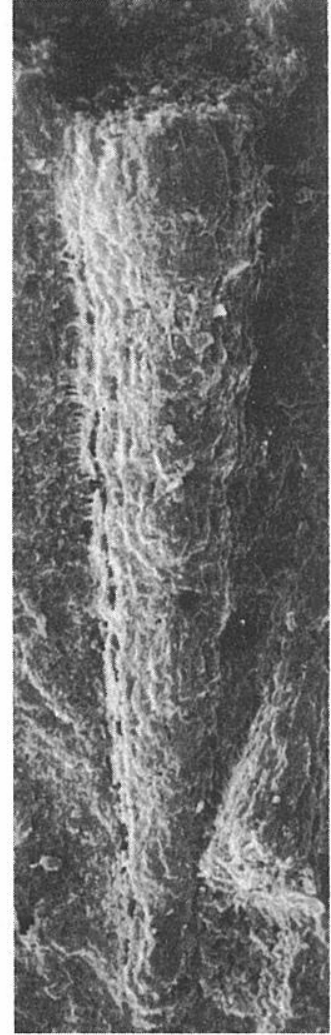

7

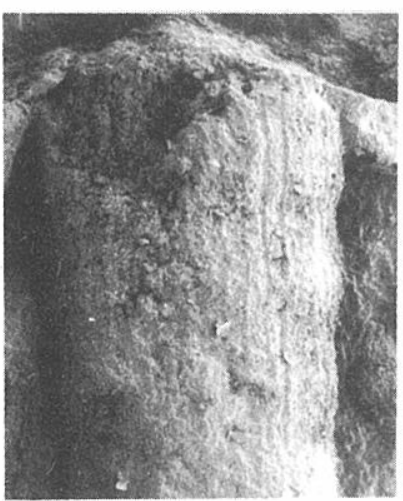

2

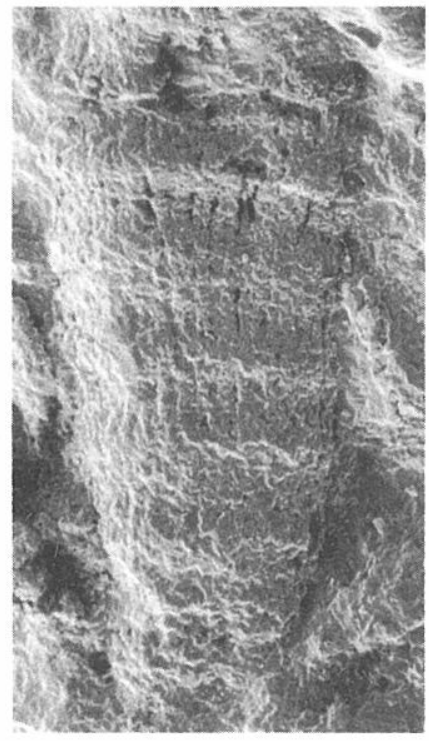

6

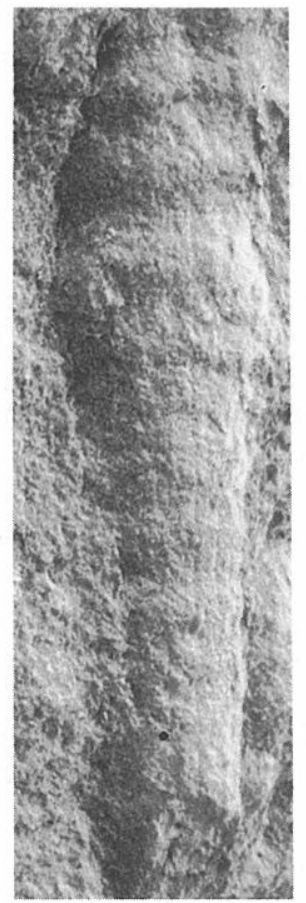

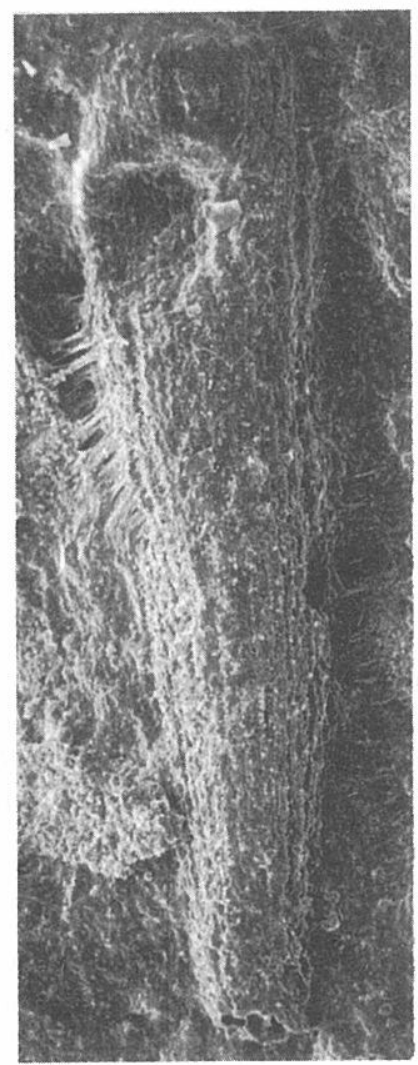

3

9

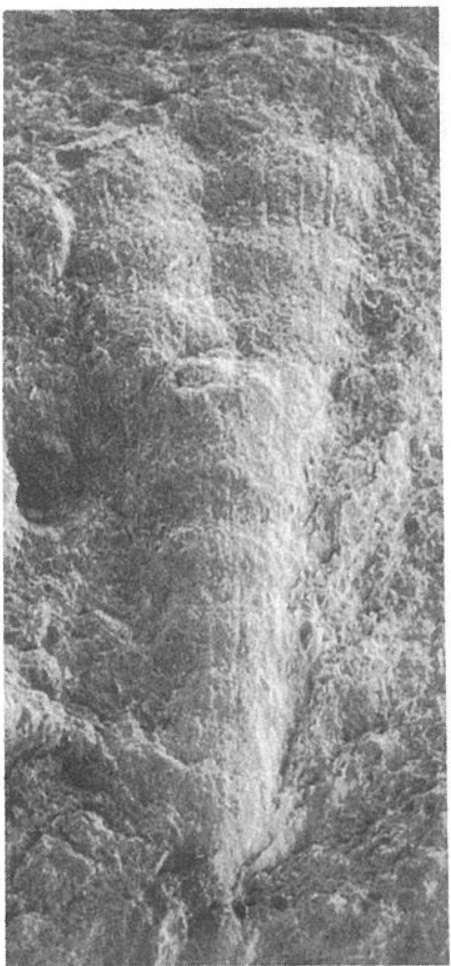

Lámina I

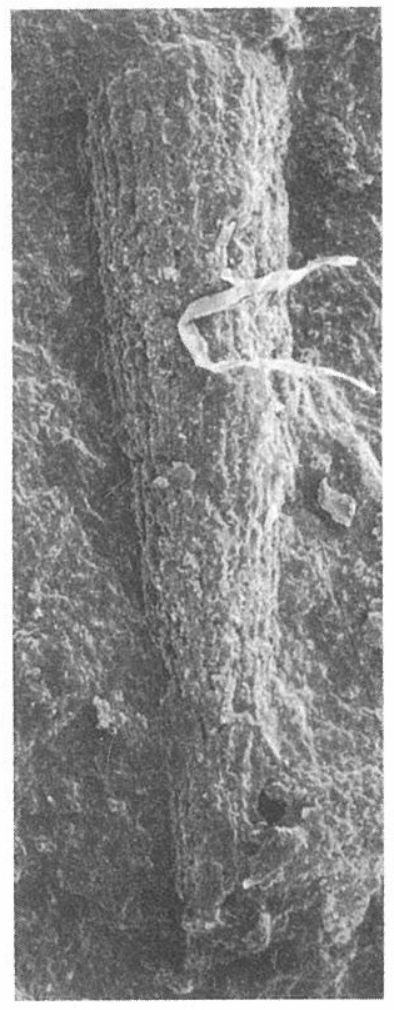

4

10
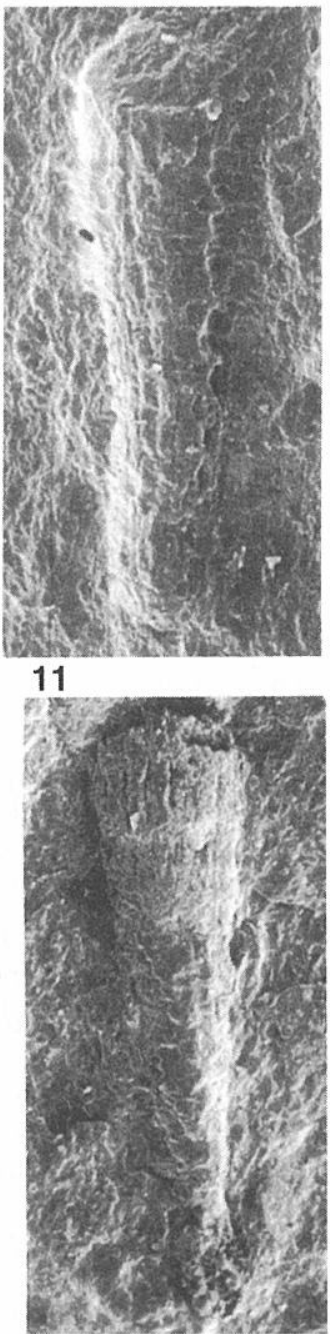

Revista Española de Paleontología, nº extr. Homenaje al Prof. J. Truyols, 1999. 
región tipo. Su hallazgo en otras áreas podría permitir el establecimiento de un entramado sólido de relaciones filogenéticas, especie a especie, entre Undastriatostyliolina y otras formas de la Familia Undastriatostyliolinidae, con los géneros Viriatellina, Striatostyliolina y, tal vez, Metastyliolina.

\section{Undastriatostyliolina globicellata Farsan,1983 Lám. I, figs. 8, 10, 11}

* 1983 Undastriatostyliolina globicellata Farsan, 39, pl. 3, figs. 14-18.

v 1985 Striatostyliolina sp.; Henn, 75.

Material y yacimientos: 9 ejemplares casi completos, 3 de ellos pertenecientes a la Sección "Gleithang" de Henn (1985, p.75), nivel 43, y media docena en estado de moldes, provenientes de la sucesión del río Carrión, nivel CAR1/2(2), todos ellos de la Fm. Cardaño, en el Dominio Palentino (Fig. 1C).

\section{Descripción}

Conchas de pequeño tamaño, de aspecto general cónico ya que el ángulo de crecimiento disminuye sólo muy ligeramente hacia la mitad de la concha. Longitud máxima medida: $1 \mathrm{~mm}$; máxima anchura: $0,33 \mathrm{~mm}$. Ángulo de crecimiento en torno a $10-11^{\circ}$. Cámara inicial redondeada, bastante grande con relación al tamaño total de la concha, separada por un débil estrangulamiento del resto; longitud entre 0,13 y $0,16 \mathrm{~mm}$ y anchura oscilando entre un valor mínimo de $0,13 \mathrm{~mm}$ y uno máximo de 0,14 .

Ornamentación transversal de ondulaciones en forma de tenues arrugas, casi imperceptibles en algún ejemplar, de distribución bastante regular, en número de 4 a 5 por medio milímetro de concha, apareciendo inmediatamente sobre la cámara inicial. Espacios interanulares mal definidos.

Ornamentación longitudinal constituida por estrías bien marcadas, moderadamente profundas, que surcan toda la concha, incluida la cámara inicial, alcanzando un número de 6 a 7 por semicircunferencia en la apertura.

\section{Discusión}

U. globicellata era conocida únicamente en su localidad tipo (Fm. Beaulieu, Boulonnais, N de Francia), en capas de edad Frasniense (Farsan, 1983). Nuestros ejemplares provienen de niveles givetienses de la Fm. Cardaño (sección "Gleithang" de Henn, 1985 y sucesión del río Carrión). No obstante, aún apareciendo en capas más antiguas, nuestros ejemplares no se diferencian del material de Farsan (1983), con conchas de tamaño similar que muestran una gruesa y redondeada cámara inicial y elementos transversales comparables en morfología y densidad; únicamente las estrías longitudinales están más profundamente marcadas sobre la concha que en las formas frasnienses, pero su distribución a lo largo de la misma desde el ápice, así como su densidad por semicircunferencia apertural, es idéntica. La menor profundización de los elementos longitudinales sobre la concha en los ejemplares procedentes del Boulonnais puede ser debida a un problema de conservación como consecuencia de su extracción, en el proceso de lavado, de la matriz margosa encajante.

Henn (1985, p.75) había señalado la presencia de Striatostyliolina en los niveles 39 y 43 de la sección "Gleithang". La mayoría de ejemplares del nivel 43 son, en efecto, formas del género Striatostyliolina, pero tres de ellos han de ser incorporados al género Undastriatostyliolina por la presencia de elementos transversales y, concretamente, por su morfología general, comparable a la especie discutida.

Por otro lado, los ejemplares del nivel 39 pertenecen también a Undastriatostyliolina, aunque seguramente a una especie distinta, Undastriatostyliolina truyolsi n. sp. (ver discusión de esta especie).

Aunque las diferencias entre las especies asignadas a este género por Farsan (1983): U. globicellata Farsan, $U$. crassa Farsan, $U$. concavimarginata Farsan y $U$. curvimarginata Farsan, son muy sutiles, la primera de ellas es la mejor individualizada y se diferencia de las otras en el número más elevado de estrías longitudinales y en el ángulo de crecimiento más abierto.

\section{Edad}

El material original de la especie procede del $\mathrm{Mb}$. de Pâtures de la Fm. Beaulieu en el Boulonnais (N de Francia), en capas del Frasniense pertenecientes a la Biozona de conodontos de Po. asymmetricus Media (Farsan, 1983; Brice, 1988).

En la Cordillera Cantábrica, U.globicellata fue encontrada en los niveles basales de la Fm. Cardaño, asociada con faunas de edad Givetiense Superior dentro de la Biozona de conodontos de Po. varcus ( $c f$. Henn, 1985 y García-Alcalde et al., 1990a) y por debajo de capas con Schindewolfoceras alcaldei, especie que aparece en un intervalo estratigráfico situado entre las biozonas de conodontos de Sch. hermanni-P. cristatus y Po. asymmetricus Más Baja, según Montesinos y Henn (1986).

\section{Undastriatostyliolina truyolsi $\mathrm{n} . \mathrm{sp}$.} Lám. I, figs. 3-5

v 1985 Viriatellina sp.; Henn, 75.

v 1985 Striatostyliolina sp.; Henn, 75.

Derivatio nominis: Especie dedicada a mi padre, Jaime Truyols, mi maestro, mi amigo.

Material y yacimientos: 9 ejemplares en estado de moldes, procedentes de la Fm. Cardaño en la sección "Gleithang" de Henn (1985, p.75); 6 ejemplares del nivel 39 y 3 del 43; Dominio Palentino.

Holotipo: Ejemplar figurado en Lám. I, fig. 5, conservado como un molde externo casi completo, DPO 34081.

Paratipos: Ejemplares figurados en Lám. I, figs. 3 (DPO 34082) y 4 (DPO 34083), procedentes de la sección Gleithang (Henn, 1985, p. 75), nivel 39. 
Localidad tipo: Sección Gleithang (Henn, 1985, p. 74, 75), situada unos $600 \mathrm{~m}$ al $\mathrm{NO}$ de la confluencia del arroyo de El Calero con el río Carrión (ENE de Vidrieros, provincia de Palencia).

Estrato tipo: nivel 43 de la sección "Gleithang" (Henn, 1985, p. 75). Tramo lutítico entre calizas arcillosas.

\section{Diagnosis}

Conchas cónicas de muy pequeño tamaño, con ángulo de crecimiento entre 10 y $11^{\circ}$. Ornamentación transversal constituida por ondulaciones muy tenuemente marcadas, en número de 4 a 5 por medio milímetro de concha, separadas por espacios interanulares mal delimitados. Estrías longitudinales bien marcadas en número de 10 a 14 por semicircunferencia.

Very small conical shells with growth angle between 10 and $11^{\circ}$. Very faint transverse rings, 4 to 5 rings per $0.5 \mathrm{~mm}$ of shell lenght. Well marked longitudinal striation. 10 to 14 longitudinal elements on the semicircunference.

\section{Descripción}

Conchas de pequeño tamaño, casi cónicas debido a la ligera disminución del ángulo de crecimiento en el tercio distal de la concha. Longitud máxima medida: $1,4 \mathrm{~mm}$ correspondiente a un ejemplar que carece de cámara inicial; máxima anchura: $0,33 \mathrm{~mm}$. Ángulo de crecimiento entre 10 y $11^{\circ}$. Cámara inicial conservada en un solo ejemplar, en forma de gota, separada de la región juvenil por un ligero estrangulamiento distal; longitud: $0,16 \mathrm{~mm}$, anchura: 0,12 $\mathrm{mm}$. Dimensiones del holotipo: Longitud: $1,2 \mathrm{~mm}$; Anchura: $0,28 \mathrm{~mm}$.

Ornamentación transversal consistente en ondulaciones muy poco marcadas, en ocasiones casi imperceptibles, pero presentes a lo largo de toda la concha. Existe una cierta variabilidad en cuanto al grado de desarrollo de los elementos transversales; en los ejemplares con ondulaciones más apreciables, se cuentan 4 a 5 por medio milímetro, regularmente distribuidas y simétricas, que se inician sobre la cámara inicial. Los espacios entre las ondulaciones están mal individualizados.

Ornamentación longitudinal formada por estrías bien marcadas, bastante profundas, que discurren ininterrumpidamente a lo largo de toda la concha, incluida la cámara incial, en número de 10 a 14 por semicircunferencia en la apertura.

\section{Discusión}

Por la densidad de estrías longitudinales y el valor del ángulo de crecimiento, Undastriatostyliolina truyolsi n. sp. se separa de todas las especies conocidas del género. Todas ellas son formas de pequeño tamaño (salvo Undastriatostyliolina n. sp. 1), con ondulaciones leves de densidad similar en todas las conchas, de modo que los criterios fundamentales para diferenciarlas, tal como indica Farsan (1983, Tabl. 2), son precisamente el número de estrías longitudinales y el valor del ángulo de crecimiento, aún cuando este autor toma también en consideración la forma de las regiones proximal y distal de la concha.
$U$. curvimarginata, U. crassa, U. globicellata y $U$. concavimarginata tienen un ángulo de crecimiento más bajo y un número de estrías netamente inferior al de Undastriatostyliolina truyolsi $\mathrm{n}$. sp. Por otro lado, Undastriatostyliolina $\mathrm{n}$. sp. 1 es de tamaño mayor, muestra ondulaciones bien marcadas, mayor número de estrías y ángulo de crecimiento más abierto.

Parte de las formas citadas por Henn (1985, p. 75) como Viriatellina sp. y Striatostyliolina sp., que hemos podido estudiar personalmente, pertenecen a Undastriatostyliolina truyolsi n. sp. En concreto, las del nivel 39 de la sección "Gleithang" y algunas de las del nivel 43.

\section{Edad}

Undastriatostyliolina truyolsi n. sp. proviene de la sección "Gleithang" en la Fm. Cardaño, dentro del Dominio Palentino, en niveles pertenecientes a la Biozona de conodontos de Po. varcus del Givetiense Superior, según datos de Henn (1985, p. 75).

\section{Undastriatostyliolina n. sp. 1 Lám. I, figs. 1, 2}

Material y yacimientos: 1 ejemplar casi completo (DPO 34077), careciendo de cámara inicial, procedente del nivel AR-47bis de la Fm. Abadía en la sucesión del río Arauz, Dominio Palentino (Fig. 1B).

\section{Descripción}

Concha de tamaño medio, casi cónica debido a la escasa disminución del ángulo de crecimiento hacia la parte media. Máxima longitud medida: $2,8 \mathrm{~mm}$ sin la cámara inicial; anchura máxima: 0,6 mm. Ángulo de crecimiento en torno a los $14^{\circ}$. Cámara inicial no conservada.

Ornamentación transversal constituida por suaves ondulaciones de perfil simétrico, que muestran una gran regularidad en su distribución, en número de 5 por milímetro. En la región juvenil son muy tenues, aumentando discretamente el relieve hacia la apertura. Espacios interanulares mal definidos, ocupando una extensión algo menor que los anillos.

Ornamentación longitudinal de estrías poco profundas pero bien marcadas, que cubren toda la concha visible del ejemplar disponible, en número de 15 a 16 por semicircunferencia en la apertura.

\section{Discusión}

El ejemplar cantábrico se diferencia claramente de las especies de Undastriatostyliolina conocidas en la actualidad. En efecto, su tamaño es muy superior, casi el doble, al de todas las especies de Farsan (1983), muestra ondulaciones más elevadas y regulares que todas ellas y, por último, un número de estrías casi dos veces superior al de dichos taxones. Desgraciadamente, por el momento solo se dispone de un único ejemplar, lo que obliga a mantener ciertas reservas y considerarlo, hasta el hallazgo de nuevo material, en nomenclatura abierta. No obstante, merece la pena destacar que sus caracteres generales, 
mayor tamaño de la concha, anillos más pronunciados y elevado número de estrías, con respecto a las especies más modernas, son primitivos dentro del grupo y coinciden con las tendencias generales que parecen mostrar diversas líneas de dacrioconáridos, con una disminución progresiva del tamaño de las conchas, del número de elementos longitudinales y la aminoración del relieve de la ornamentación transversal.

\section{Edad}

El ejemplar ha sido encontrado en la Fm. Abadía (sucesión del río Arauz, Fig. 1B), en un nivel perteneciente a la Biozona de dacrioconáridos de $N$. cancellata del Emsiense superior (Truyols-Massoni, 1988), equivalente o muy próximo a capas que han suministrado a Montesinos (1991) Mimagoniatites ex gr. fecundus.

\section{Undastriatostyliolina n. sp. 3 Lám. I, figs. 6, 7, 9}

Material y yacimientos: Varios ejemplares fragmentados, procedentes del nivel CAR-3/4(1) de la Fm. Cardaño, en la sucesión del río Carrión, Dominio Palentino (Fig. 1C).

\section{Descripción}

Conchas pequeñas, cónicas en la región juvenil, adquiriendo aspecto subcilíndrico hacia la parte media debido a la disminución de su ángulo de crecimiento. Máxima longitud medida: $1,2 \mathrm{~mm}$, correspondiendo a un ejemplar que carece de cámara inicial; anchura máxima: $0,30 \mathrm{~mm}$. Ángulo de crecimiento en torno a los $13^{\circ}$. Cámara inicial no conservada.

Ornamentación transversal consistente en ondulaciones suaves pero bien marcadas, de perfil simétrico, regularmente distribuidas a lo largo de toda la concha. Su densidad es de 8-9 elementos por milímetro. Espacios interanulares no demasiado bien delimitados, cóncavos, ocupando una extensión igual o ligeramente inferior que los anillos.

Ornamentación longitudinal de estrías finas, que discurren ininterrumpidamente a lo largo de toda la concha, en número de 12 a 16 por semicircunferencia en la apertura. Se desconoce si estos elementos recubrían o no la cámara inicial.

\section{Discusión}

Undastriatostyliolina $\mathrm{n}$. sp. 3 se diferencia netamente de todas las especies descritas por Farsan (1983), procedentes de la Fm. Beaulieu (Boulonnais, $\mathrm{N}$ de Francia), así como de Undastriatostyliolina n. sp. 1, del Dominio Palentino. Undastriatostyliolina truyolsi $\mathrm{n}$. $\mathrm{sp}$. es una forma próxima a Undastriatostyliolina $\mathrm{n}$. sp. 3, pero su concha es más cónica, tiene un ángulo de crecimiento más cerrado, ondulaciones transversales mucho más débiles y en número muy inferior, y estrías más profundas en número ligeramente menor.

\section{Edad}

El material procede del nivel CAR-3/4(1) de la Fm. Cardaño en la sucesión del río Carrión (Fig. 1C), de edad
Givetiense Superior, situado por debajo de capas con Schindewolfoceras (nivel CAR-7/8(3)) (Montesinos, com. pers.), probablemente equivalentes a los niveles 28,30 y 3 de Cardaño I en Montesinos y Henn (1986), que han suministrado Schindewolfoceras alcaldei. Este tramo con $S$. alcalde $i$ pertenece a un intervalo estratigráfico situado entre las Biozonas de Sch. hermanni-P cristatus y Po. asymmetricus Más Baja con base en los conodontos determinados por Henn (1985).

\section{AGRADECIMIENTOS}

La autora agradece las minuciosas correcciones de los dos revisores, $\mathrm{M}^{\mathrm{a}}$ Luisa Martínez Chacón y Miguel Pardo, que han mejorado, sin duda, el conjunto del manuscrito. Este trabajo se ha realizado en el marco del Proyecto DG 941324 y es una contribución al Programa Internacional de Correlación Geológica n 421.

\section{BIBLIOGRAFÍA}

Arbizu, M.A. 1978. Trilobites Synphoriidae del Devónico de la Cordillera Cantábrica: Malladaiinae nov. subfam. Trabajos de Geología, Universidad de Oviedo, 10, $37-$ 65.

Bergström, S.M. 1990. Relations between conodont provincialism and the changing palaeogeography during the Early Palaeozoic. In: Palaeozoic Palaeogeography and Biogeography (Eds. W.S. McKerrow and C.R. Scotese). Geological Society Memoir 12, 105-121.

Bouyx, E., Blaise, J., Brice, D., Gourvennec, R., Lardeux, H. et Le Menn, J. 1992. Implications paléogéographiques des affinités nordgondwaniennes et rhénanes des faunes dévoniennes de la zone de Meguma (Appalaches septentrionales). Comptes Rendus de l'Academie des Sciences, Paris, 315, II, 337-343.

Brice, D. 1988. Le Dévonien de Ferques (BoulonnaisFrance). Historique. Synthèse des données nouvelles et stratigraphie, sedimentologie, paléontologie et tectonique. Conclusions. In: Le Dévonien de Ferques. Bas-Boulonnais (N. France) (Ed. D. Brice). Biostratigraphie du Paléozoïque, 7, 7-24.

Brice, D., Milhau, B. et Mistiaen, B. 1994. Affinités nordaméricaines de taxons dévoniens (Givetien-Frasnien) du Boulonnais, Nord de la France. Migrations et diachronismes. Bulletin de la Société Géologique de France, 165 (4), 291-306.

Farsan, N.M. 1983. Tentaculites du Frasnien Inférieur de Ferques (Boulonnais, Nord de la France). Palaeontographica, 182 (1-3), 26-43.

Farsan, N.M. 1994. Tentaculiten: Ontogenese, Systematik, Phylogenese, Biostratinomie und Morphologie. Abhandlungen Senckenbergischen Naturforschenden Gesellschaft, 547, 1-128.

Frankenfeld, H. 1983. El Manto de Montó-Arauz: interpretación estructural de la Región del PisuergaCarrión (Zona Cantábrica, España). Trabajos de Geología, Universidad de Oviedo, 13, 37-47. 
García-Alcalde, J.L. 1993. Océanos y mares paleozoicos al NO de Gondwana. Paleogeografía de la Zona Cantábrica. Revista Española de Paleontología, 8, 132.

García-Alcalde, J.L. 1995. L'évolution paléogéographique pré-varisque de la Zone Cantabrique septentrionale (Espagne). Revista Española de Paleontología, 10, 9-29.

García-Alcalde, J.L. and Truyols-Massoni, M. 1994. Lower/Upper Emsian versus Zlichovian/Dalejian (Lower Devonian) boundary. Newsletter on Stratigraphy, 30, 8389.

García-Alcalde, J.L., Montesinos, J.R., Truyols-Massoni, M., García-López, S., Arbizu, M.A. and Soto, F. 1990a. The Palentine Domain (Palentian Zone). In: PreMesozoic Geology of Iberia (Eds. R.D. Dallmeyer and E. Martínez García). Springer-Verlag, Berlin-Heidelberg, 20-23.

García-Alcalde, J.L., Arbizu, M.A., García-López, S., Leyva, F., Montesinos, J.R., Soto, F. and TruyolsMassoni, M. 1990b. Devonian Stage boundaries (Lochkovian/Pragian, Pragian/Emsian and Eifelian/ Givetian) in the Cantabric region (NW Spain). Neues Jahrbuch Geologische und Paläontologische Abhandlungen, 180, 177-207.

García-López, S., Julivert, M., Soldevila, M., TruyolsMassoni, M. y Zamarreño, I. 1990. Biostratigrafía y Facies de la sucesión carbonatada del Silúrico Superior y Devónico Inferior de Santa Creu d'Olorda (Cadenas Costero Catalanas, NE de España). Acta Geologica Hispanica, 25, 141-168.

Henn, A.H. 1985. Biostratigraphie und Fazies des hohen Unter-Devon bis tiefen Ober-Devon der Provinz Palencia, Kantabrisches Gebirge, N-Spanien. Göttinger Arbeiten zur Geologie und Paläontologie, 26, 1-100.

Jahnke, H., Henn, A., Mader, H. und Schweineberg, J. 1983. Silur und Devon im Arauz-Gebiet (Prov. Palencia, NSpanien). Newsletter on Stratigraphy, 13, 40-66.

Julivert, M., Fontboté, J.M., Ribeiro, A. y Conde, L.E. 1972. Mapa Tectónico de la Península Ibérica y Baleares E. 1: 1.000.000. Instituto Geológico y Minero de España, Madrid, 1-113.

Kullmann, J. 1960. Die Ammonoidea des Devon im Kantabrischen Gebirge (Nordspanien). Akademie der Wissenschaften und der Literatur in Mainz, 7, 457-559.

Lütke, F. 1979. Biostratigraphical significance of the Devonian Dacryoconarida. In: The Devonian System (Eds. M.R. House, C.T. Scrutton and M.G. Bassett). Special papers on Paleontology, 23, 281-291.

Lütke, F. 1985. Devonian tentaculites from Nevada (U.S.A.). Courier Forschungs-Institut Senckenberg, 75, 197-226.

Marquínez, J.L. y Marcos, A. 1984. La estructura de la Unidad del Gildar-Montó (Cordillera Cantábrica). Trabajos de Geología, Universidad de Oviedo, 14, 5364.

Montesinos, J.R. 1991. Ammonoideos de las Capas de Vañes (Formación Abadía, Devónico Inferior) del Dominio Palentino (Palencia, NO de España). Cuadernos do Laboratorio Xeolóxico de Laxe, 16, 193201.

Montesinos, J.R. y Henn, A.H. 1986. La Fauna de
Pharciceras (Ammonoidea) de la Formación Cardaño (Dominio Palentino, Cordillera Cantábrica, NO de España). Trabajos de Geología, Universidad de Oviedo, 16, 61-76.

Montesinos, J.R. y Sanz López, J. 1997. Los ammonoideos de la parte superior de la Formación Cardaño (Unidad del Gildar-Montó, NE de la provincia de León, España) y su aplicación a la bioestratigrafía del Devónico Superior. Revista Española de Paleontología, 12, 243248.

Montesinos López, R. y Truyols-Massoni, M. 1987. La Fauna de Anetoceras en el Dominio Palentino y el limite Zlichoviense/Dalejiense (Palencia, N. de España). Cuadernos do Laboratorio Xeolóxico de Laxe, 11, 191208.

Morzadec, P., Paris, F., Plusquellec, Y. and Racheboeuf, P.R. 1988. Devonian stratigraphy and paleogeography of the Armorican Massif (Western France). In: Devonian of the World (Eds. N.J. Mc.Millan, A.F. Embry and D.J. Glass) Proceedings 2nd. International Symposium Devonian System. Canadian Society Petroleum Geology, Memoirs, 14, 401-420.

Racheboeuf, P.R. 1981. Chonétacés (Brachiopodes) siluriens et dévoniens du Sud-Ouest de 1'Europe. Mémoires de la Société Géologique et Minéralogique de Bretagne, 27, 1294.

Rodríguez Fernández, L.R. and Heredia, N. 1990. Palentian Zone. In: Pre-Mesozoic Geology of Iberia (Eds. R.D. Dallmeyer and E. Martínez García). Springer-Verlag, Berlin-Heidelberg, 69-71.

Scotese, C.R. and McKerrow, W.S. 1990. Revised world maps and introduction. Memoir of the Geological Society, 12, 1-21.

Soto, F.M. 1979. Considérations paléobiogéographiques sur les Streptelasmatina (Coelenterata, Rugosa) solitaires du Dévonien des Monts Cantabriques (NW de l'Espagne). Geobios, 12, 399-409.

Truyols-Massoni, M. 1981a. Primera aportación sobre los Tentaculites Dacrioconáridos de la región del PisuergaCarrión (Palencia, España). Revista Española de Micropaleontología, 13, 69-88.

Truyols-Massoni, M. 1981b. Sobre la Fauna de Dacrioconáridos del Grupo La Vid (Devónico Inferior, Cordillera Cantábrica, NO de España). Breviora Geologica Asturica, 25 (3/4), 25-30.

Truyols-Massoni, M. 1986. Nowakia acuaria (Praguiense, Devónico Inferior) de la Zona Asturoccidental-leonesa (NO de España). Breviora Geologica Asturica, 27 (1/2), 12-16.

Truyols-Massoni, M. 1988. Dacrioconáridos y Homocténidos del Devónico de España (Cordillera Cantábrica y otras áreas). Tesis Doctoral, Universidad de Oviedo (inédita), 1-269.

Truyols-Massoni, M. (en prensa). La edad de las Capas con Mimosphinctes en el Devónico de la Cordillera Cantábrica (NW de España). Trabajos de Geología, Universidad de Oviedo, vol. extraordinario.

Manuscrito recibido: 31 de enero, 1998 Manuscrito aceptado: 23 de marzo, 1999 